Informe especial

\title{
Reunión de trabajo sobre la medición de la presión arterial: recomendaciones para estudios de población
}

\author{
Iniciativa Panamericana sobre la Hipertensión ${ }^{1}$
}

RESUMEN Como parte de la Iniciativa Panamericana sobre la Hipertensión Arterial, la Organización Panamericana de la Salud y el Instituto Nacional del Corazón, los Pulmones y la Sangre, uno de los Institutos Nacionales de Salud de los Estados Unidos de América, llevaron a cabo una reunión de trabajo para examinar los métodos de medición de la presión arterial (PA) empleados en estudios de prevalencia y ensayos clínicos sobre la hipertensión arterial. El objetivo era desarrollar un protocolo para la medición de la PA que pudiera usarse en los estudios de prevalencia de hipertensión arterial que se realizan en las Américas. Ningún protocolo común de este tipo ha existido antes en la Región, y debido a ellos ha sido difícil comparar las estrategias de intervención y prevención en torno a la hipertensión arterial. Este artículo describe un método estándar que se ha propuesto para medir la PA en estudios de población en la Región de las Américas. El artículo examina todo lo que encierra la elaboración de un protocolo común para medir la PA, aspectos críticos de la medición de la PA en estudios nacionales, los procedimientos mínimos para la medición de la PA durante actividades de vigilancia y la evaluación de la calidad de la PA.

Palabras clave Presión sanguínea, determinación de la presión sanguínea, hipertensión, pautas, vigilancia de la población.

Los días 12 y 13 de junio de 2000, el Instituto Nacional del Corazón, los Pulmones y la Sangre (National Heart, Lung, and Blood Institute) y la Organización Panamericana de la Salud llevaron a cabo una reunión de trabajo para discutir los métodos de medición de la presión arterial (PA) empleados en encuestas de prevalencia y ensayos clíni-

\footnotetext{
Pan Americana Hypertension Initiative, Executive Secretariat, c/o Edward J. Roccella, National High Blood Pressure Education Program, National Heart, Lung, and Blood Institute, 31 Center Drive MSC 2480, Bethesda, Maryland 20892-2480, United States of America. Correo electrónico: roccella@ nih.gov.
}

cos de hipertensión arterial. Este taller es parte de la Iniciativa Panamericana sobre la Hipertensión Arterial (1). El objetivo de la reunión de trabajo fue elaborar un protocolo para la medición de la PA que pueda utilizarse en estudios sobre la prevalencia de la hipertensión arterial en las Américas. No ha habido nunca ningún protocolo común de este tipo en la Región, de tal manera que ha resultado difícil comparar las distintas estrategias de intervención y prevención de la hipertensión cuando las mediciones de la PA han sido obtenidas mediante diferentes procedimientos. Por ejemplo, algu- nos estudios sobre la prevalencia de PA definen la hipertensión a partir de 160/95 mm Hg, mientras que otros utilizan 140/90 mm Hg. Los estudios en general definen la presión diastólica como el quinto ruido de Korotkov (K5) escuchado, pero en algunos estudios se especifica que el criterio es la desaparición del ruido, mientras que en otros el criterio es el inicio del silencio, que es $2 \mathrm{~mm} \mathrm{Hg}$ por debajo de K5. Además, los estudios difieren en cuanto al número de mediciones de la PA realizadas, el instrumental empleado, los métodos de entrenamiento y los procedimientos de verificación. 


\section{CONSIDERACIONES EN TORNO A LA ELABORACIÓN DE UN PROTOCOLO COMÚN PARA MEDIR LA PRESIÓN ARTERIAL}

Las diferencias en la definición de la PA y en los criterios de medición pueden generar diferencias sustanciales en la prevalencia de la hipertensión arterial. Por lo tanto, los datos no pueden ser comparados ni combinados, y debido a ello se pierden las ventajas de examinar poblaciones con muestras muy grandes. Además, esto impide que los cambios o las mejorías en las tasas de control de la hipertensión o en los valores medios de PA se cuantifiquen a lo largo del tiempo. Por lo tanto, los programas y actividades de intervención comunitarios no se pueden evaluar. Por añadidura, es importante enseñarle al personal técnico a calibrar adecuadamente los equipos, a usar un manguito del tamaño adecuado y a evitar la preferencia de determinados valores durante la medición.

\section{ASPECTOS CRÍTICOS ACERCA DE LA MEDICIÓN DE LA PRESIÓN ARTERIAL EN ESTUDIOS NACIONALES}

Debido a que la PA puede variar en una misma persona en un lapso breve, es necesario medirla varias veces por lo menos en una ocasión. La PA debe medirse tres veces en una sesión, preferiblemente antes de un interrogatorio minucioso o un procedimiento, como la extracción de sangre, que también podrían ser parte de la encuesta.

Se debe emplear un esfigmomanómetro de mercurio estándar o un dispositivo automático certificado para medir la PA. Los esfigmomanómetros de mercurio han sido los dispositivos más usados para medir la PA, a pesar de que los están retirando de Europa y los Estados Unidos de América debido a inquietudes de tipo ambiental relacionadas con la posibilidad de que de un instrumento roto se derrame mercurio y haya que limpiarlo, cosa que es sumamente cara (2). Los dispositivos automáticos podrían llegar a usarse más en los estudios de vigilancia de la presión arterial y son los que se utilizan en la actualidad. Sin embargo, deben ser calibrados contra un estándar de mercurio o un medidor digital de presión validado, y deben satisfacer los estándares de la Sociedad Británica para el Estudio de la Hipertensión (3) o los de la Asociación para el Avance de la Instrumentación Médica (4). Los dispositivos diseñados para medir la PA en los dedos o en la muñeca son inadecuados para los estudios de vigilancia. Los esfigmomanómetros aneroides son menos confiables, pero, cuando se usan, tienen que calibrarse con frecuencia (5).

Los técnicos deben ser entrenados, certificados al inicio y certificados nuevamente con periodicidad en lo que respecta a los procedimientos para medir la PA y cuidar del instrumental. Debe haber disponibilidad de manguitos de diferentes tamaños, hasta cuatro en algunos casos. Si hace falta un estetoscopio, debe utilizarse un modelo con campana. Se debe aplicar un breve cuestionario para determinar si los participantes del estudio han recibido anteriormente un diagnóstico de hipertensión y si toman medicamentos antihipertensivos en el momento de la investigación. Para garantizar que las mediciones sigan teniendo la misma calidad, los técnicos deben ser evaluados con regularidad por supervisores y la calidad de su trabajo debe ser avalada periódicamente mediante tablas estadísticas para detectar sesgos en las mediciones. Puede ser necesario volver a entrenar al personal técnico para que no pierda las habilidades adquiridas.

\section{PROCEDIMIENTOS MÍNIMOS PARA LA MEDICIÓN DE LA PRESIÓN ARTERIAL DURANTE LA VIGILANCIA}

Los procedimientos para lograr una medición exacta de la PA son los siguientes:

1. El participante debe sentarse a una mesa sosegadamente, con ambos pies apoyados totalmente sobre el suelo y con la espalda contra un respaldo. La vejiga debe estar vacía. La habitación debe ser cómoda y poco ruidosa. No se deben haber consumido bebidas alcohólicas ni productos a base de tabaco ni cafeína durante los 30 minutos previos a la medición. $\mathrm{Si}$ esto no es posible, debe constar entre los datos anotados.

2. El brazo derecho, que debe estar desnudo, se coloca sobre la mesa (al nivel del corazón) ligeramente flexionado, con la palma de la mano hacia arriba. El investigador debe estar en una posición que le permita ver el manómetro a la altura de sus ojos.

3. Determine la circunferencia del brazo y escoja y coloque un manguito de tamaño adecuado. El borde inferior del manguito debe estar $2,5 \mathrm{~cm}$ por encima de la articulación del codo.

4. Espere 5 minutos.

5. Palpe el pulso radial e infle el manguito hasta llegar a $30 \mathrm{~mm}$ $\mathrm{Hg}$ por encima del nivel en el que desaparece el pulso radial (nivel de máxima inflación). Desinfle el manguito.

6. Espere 30 segundos antes de volver a inflar el manguito.

7. Infle el manguito hasta llegar al nivel de máxima inflación.

8. Desinfle el manguito a $2 \mathrm{~mm} \mathrm{Hg}$ por segundo.

9. Registre la PA sistólica, la fase 1 de Korotkov (el primero de por lo menos dos ruidos regulares consecutivos). Anote el número par más cercano.

10. Registrar la PA diastólica, la fase 5 de Korotkov (el final del último ruido escuchado). Anote el número par más cercano.

11. Termine de desinflar el manguito, levante el brazo del participante por encima del nivel del corazón durante 15 segundos. Descanse un minuto y proceda a realizar la medición dos veces más. Utilice el valor medio de las últimas dos mediciones.

Cuando se utilizan dispositivos automáticos, es necesario observar los puntos 1, 2, 3, 4 y 11. El punto 5 depende de si el dispositivo automático mide la 
presión durante el inflado del manguito. Los otros puntos no son aplicables a estos dispositivos. Todos los puntos son aplicables cuando se utiliza un dispositivo manual de auscultación.

\section{EVALUACIÓN DE LA CALIDAD DE LA PRESIÓN ARTERIAL}

Antes de llevar a cabo un estudio se deben tener en cuanta los siguientes aspectos. El problema de los dígitos preferidos se asocia por lo general con los dispositivos manuales, aunque también se ha demostrado con el uso de dispositivos automáticos.

Los análisis de control de la calidad pueden identificar a los técnicos y pro- cedimientos que necesitan medir mejor la presión. Como el desinflado del manguito se hace a $2 \mathrm{~mm} \mathrm{Hg}$ por segundo y las lecturas se redondean al número par más cercano, se debe determinar si los dígitos pares son siempre uniformes. Esto se hace contando el número de mediciones observadas que terminan en cada uno de los dígitos pares y calculando un estadístico mediante la prueba de la ji al cuadrado, así como un puntaje que refleje la preferencia por cada dígito. Como se realizan como mínimo dos mediciones de PA, los técnicos que muestren diferencias extremas en estas dos mediciones o cuyas mediciones sean idénticas con más frecuencia de la esperada deben recibir un entrena- miento adicional. Puede que los técnicos no completen todas las mediciones de PA (esto puede verse en una tabla de datos incompletos), pero si esto se ve con más frecuencia en unos que en otros, entonces se necesita un mayor entrenamiento. Se deben calcular los valores promedio de PA en fechas secuenciales para determinar si hay desplazamientos (cambios repentinos) o deriva (cambios graduales) en los valores promedio a lo largo del tiempo. Esto presupone que los participantes se asemejan los unos a los otros mientras dura el estudio. Estos análisis del control de la calidad se describen detalladamente en un informe de la Organización Mundial de la Salud (6).

\section{REFERENCIAS}

1. Pan American Hypertension Initiative. Pan American Hypertension Initiative [sitio en Internet]. Disponible en: http://www.mco.edu/ org/whl/pahi.html. Acceso el 12 de septiembre de 2003.

2. United States of America, National Institutes of Health. Mad as a hatter? Join our campaign for a mercury-free $\mathrm{NIH}$ [página de Internet]. Disponible en: http://www.nih.gov/od/ors/ ds/nomercury/index.htm. Acceso el 12 de septiembre de 2003.

3. British Hypertension Society. Blood pressure monitors [página de Internet]. Disponible en: http://www.hyp.ac.uk/bhsinfo/bpmindex. $\mathrm{html}$. Acceso el 12 de septiembre de 2003.

4. Association for the Advancement of Medical Instrumentation. Association for the Advancement of Medical Instrumentation [sitio en Internet]. Disponible en: http://www. aami.org. Acceso el 12 de septiembre de 2003.

5. Canzanello VJ, Jensen PL, Schwartz GL Are aneroid sphygmomanometers accurate in hospital and clinic settings? Arch Intern Med 2001;161(5):729-731.

6. Kuulasmaa K, Hense H-W, Tolonen H. Quality assessment of data on blood pressure in the
WHO MONICA Project. Geneva: World Health Organization and the WHO MONICA Project; 1999 [sitio en Internet]. Disponible en: http://www.ktl.fi/publications/monica/ $\mathrm{bp} / \mathrm{bpqa} . \mathrm{htm}$. Acceso el 12 de septiembre de 2003.

Manuscrito recibido el 14 de agosto de 2003. Aceptado para publicación, tras revisión, el 25 de septiembre de 2003.

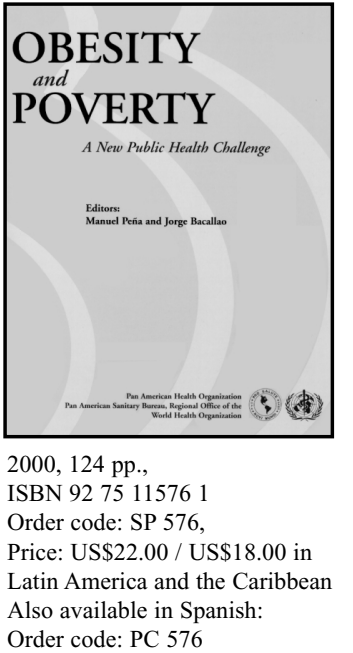

Obesity and Poverty: A New Public Health Challenge

Obesity and Poverty: A New Public Health Challenge is an essential source for understanding the new face of poverty in the Region of the Americas. This up-todate examination of the prevalence of overweight and obesity in the Region's countries looks at these conditions' medium- and long-term harmful consequences and explores their implications for planning public health interventions.

The book analyzes how the Region's countries experience the nutritional transition process that is under way worldwide, a process that is tied to the global demographic and epidemiologic transition. In this context, the increase in obesity and overweight observed in the Hemisphere coexists with a risk factor that differs from traditional risk factors seen in developed countries - the persistence of the increase in inequalities and inequities in health.

http://publications.paho.org • Fax: (301) 206-9789 • E-mail: paho@pmds.com 